\title{
Influence on sampling interval of 3D object in CGH reconstruction
}

\author{
Kimura Mizuho, Daiki Yasukochi, Takayuki Yokoyama, Yosuke Koga, Shiyuan Yang* \\ ${ }^{a}$ Kyusyu Institute of Technology, 1-1 Sensui-cho Tobata-ku Kitakyusyu-shi Fukuoka-ken, 804-8550, Japan \\ * Corresponding Author: yang@elcs.kyutech.ac.jp
}

\begin{abstract}
Computer-generated hologram $(\mathrm{CGH})$ can reconstruct the three dimensional distribution of an object. For this purpose, it is necessary to use sufficiently large hologram number of pixels (sampling points) and enough high resolution (sampling interval). However, in previous studies, the criteria for the sampling interval of the hologram and the number of sampling points necessary to reproduce an image has not been determined. For these reasons, it is necessary to determine the criteria for the sampling interval of the hologram and the sampling points. In this paper, as its basic research, we describe the effect of the reconstructed image of $3 \mathrm{D}$ object due to a change in the hologram of the sampling interval in the computer hologram.
\end{abstract}

Keywords: hologram, sampling interval, three-dimensional object.

\section{Introduction}

Hologram is the produced recording medium by holography that is a recording and reproducing technology for 3D object. Light wave front has the amplitude and phase information. However, conventional recording medium can only record the amplitude information. In contrast, the hologram can record amplitude information and phase information of the original object light. Therefore, the hologram can reproduce 3D image. Interference of light is utilized as a means to record amplitude information and phase information simultaneously. By interfering with the reference light to the object light, the hologram can record the phase information together with the amplitude information of object.

There is computer-generated hologram $(\mathrm{CGH})$ as one type of holograms. Computer-generated hologram uses the interference fringes from the distribution of diffraction from the object light and reference light by calculation. By utilizing Computer-generated hologram, creating a hologram becomes easy. There is also the advantage of creating a hologram for $3 \mathrm{D}$ object fictitious not exist in the real world. However, because data that can be handled on the computer are all discrete values, the error is caused by discretization and quantization. Thereby, the reproduced image is deteriorated. As a solution, there is a way that a sufficiently large number of discrete points. For this purpose, it is necessary to use a hologram that is sufficiently large number of pixels (sampling points). However, in previous studies, the criteria for the sampling interval of the hologram and the number of sampling points necessary to reproduce an image has not been determined. For these reasons, it is necessary to determine the criteria for the sampling interval of the hologram and the sampling points. In this paper, we describe the effect of the reconstructed image of $3 \mathrm{D}$ object due to a change in the hologram of the sampling interval in the computer hologram and examined whether it is possible to reduce the number of sampling points of the hologram.

\section{Sampling interval of computer-generated hologram}

\subsection{Generation and Reproduction of hologram}

Conventional recording medium can only record the amplitude information. However, by using an interference it is possible to record the amplitude information and phase information simultaneously. Interference fringes are generated by interference with the reference light and object light. Amplitude and phase information of the object light is recorded in the interference fringes. By recording interference fringes in hologram, hologram can be recorded amplitude and phase information of the object. When 
irradiated with reference light to the hologram, identical image as the original object is reproduced. By looking into the hologram, reconstructed image is visible in the position where there is the original object. It shows generation process and reproduction process of hologram in Figure 1 and Figure 2.

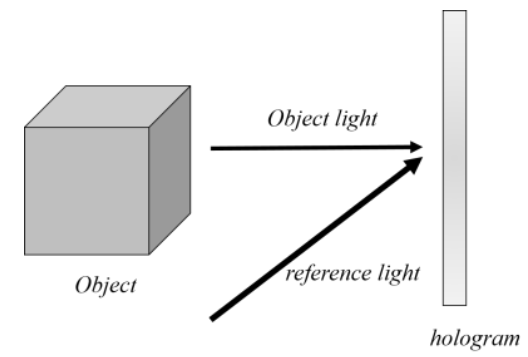

Figure 1. Generation hologram

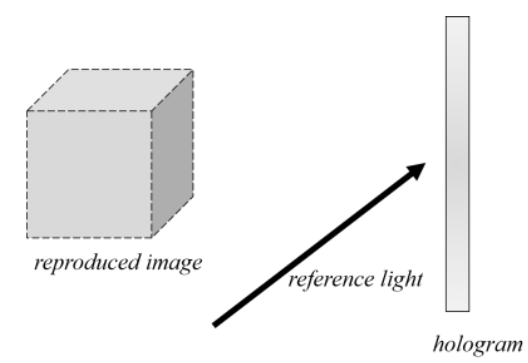

Figure 2. Reproduction hologram

\subsection{Classification of computer-generated hologram}

Computer-generated hologram is a hologram that is created on a computer. Computer-generated hologram obtains the distribution of diffraction of the object light and reference light and interference fringes by calculation. By utilizing Computer-generated hologram, creating a hologram becomes easy. There is also the advantage of creating a hologram for 3D object fictitious not be present in the real world.

The recording method of the interference fringes have an amplitude-type and a phase type. In the amplitude type to record the amplitude information of the interference fringes. In the phase-type records the phase information of the interference fringes. In this study, we used the amplitude type computer hologram.

\subsection{Sampling theorem}

Data that can be handled on a computer are all discrete values. Therefore, if you want to produce a computer-generated hologram, there is a need to deal with discrete data.
At that time, it is necessary to decide whether to use how much of the sampling points. To be considered at that time is the sampling theorem. If the interval of the sampling points becomes larger, the reproduced image because the error occurs is deteriorated. At this time, distance of the sample point for sampling is called the sampling interval

\section{Influence on reconstructed image}

\subsection{Influence of the depth of the object}

First, we examined the influence on the reconstructed image due to the depth of the object. Original image is a square distribution shown in Figure 3. Inclining the original image, as shown in Figure 4 and Figure 5. By inclining the original image, the front portion and the back of the part is made to the hologram. Therefore, depth occurs. This is regarded as a pseudo 3D objects. This depth is increased by increasing the inclination. We simulate under the following conditions.

- Original images : $64 \times 64$ [pixel]

- Hologram : 64×64[pixel]

- Pixel interval (original images): $100[\mu \mathrm{m}]$

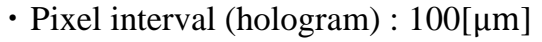

- The inclination of the original image : $0,0.01,1,10\left[^{\circ}\right]$

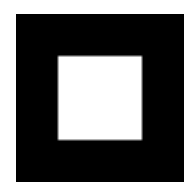

Figure 3. Original image

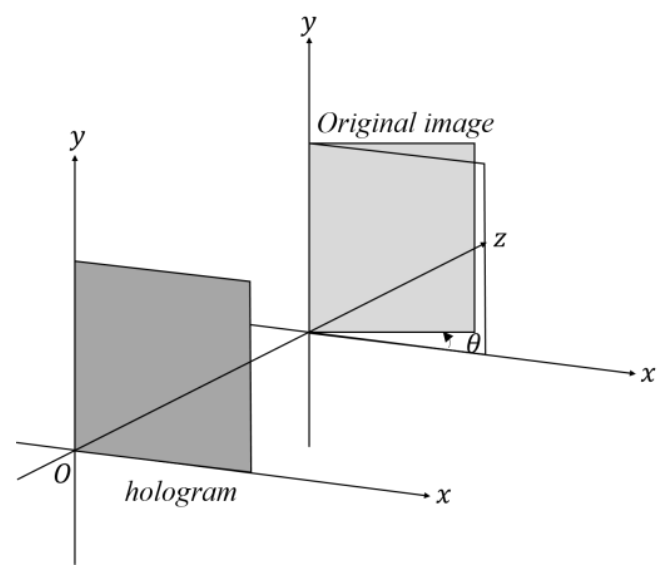

Figure 4. Inclining the original image 


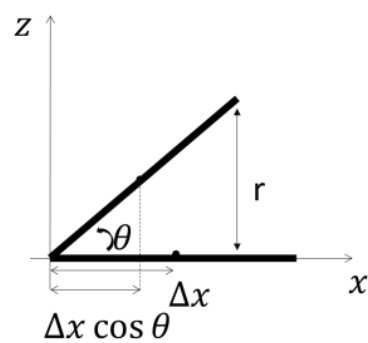

Figure 5. Inclining the original image(depth)

Figure 6 shows the simulation results. Reconstructed image is degraded larger the inclination. In other words, when the depth of the original object becomes big, reconstructed image degrades.

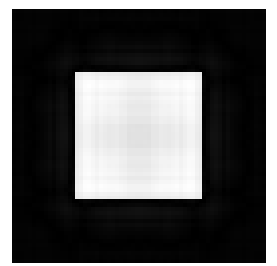

(a) $0^{\circ}$

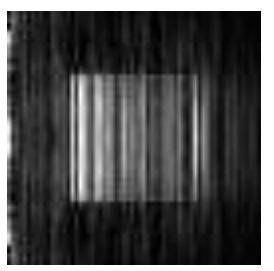

(c) $1^{\circ}$

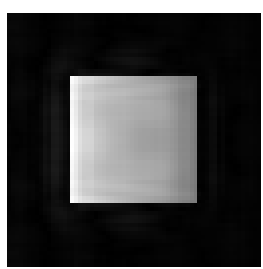

(b) $0.01^{\circ}$

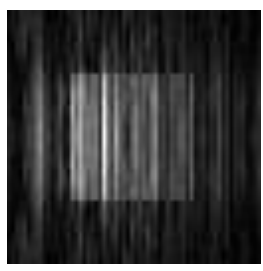

(d) $10^{\circ}$
Figure 6. Reconstruction image

(Change the depth)

\subsection{Influence of sampling points}

Next, we examined the influence of the sampling points on the reproduced image. We simulate under the following conditions.

- Original images : $64 \times 64$ [pixel]

- Hologram : 64×64, 128×128, 256×256 [pixel]

- Pixel interval (original images): $100[\mu \mathrm{m}]$

- Pixel interval (hologram) : $100[\mu \mathrm{m}]$

- The inclination of the original image : $1\left[^{\circ}\right]$

Figure 7 shows the simulation results.

Reconstructed images improve by increasing sampling points of hologram

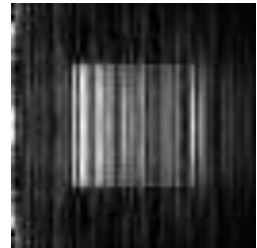

(a) $64 \times 64$

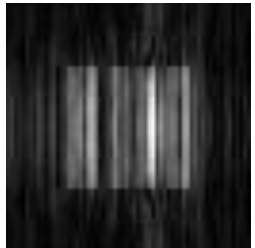

(b) $128 \times 128$

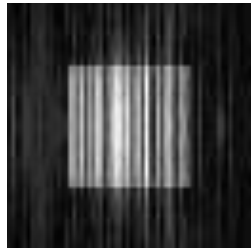

(c) $256 \times 256$
Figure 7. Reconstruction image

(Change sampling points)

\subsection{Influence of sampling interval}

Next, while fixing the number of sampling points of the hologram, changing the sampling interval. Then we examined the influence of the sampling interval on the reproduced image. We simulate under the following conditions.

- Original images : $64 \times 64$ pixel

- Hologram : $256 \times 256$ pixel

- Pixel interval (original images): $100[\mu \mathrm{m}]$

- Pixel interval (hologram) : 25, 50, 100[ $\mu \mathrm{m}]$

- The inclination of the original image : $1\left[^{\circ}\right]$

Figure 8 shows the simulation results. Reconstructed images improve by narrowing the sampling interval.

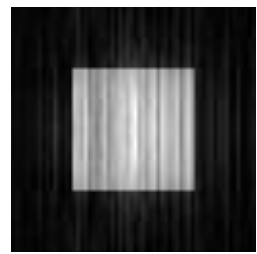

(a) $25 \mu \mathrm{m}$

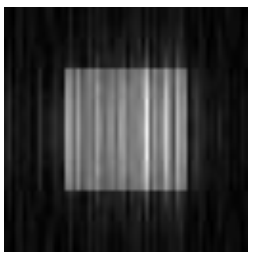

(b) $50 \mu \mathrm{m}$

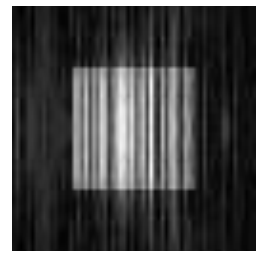

(c) $100 \mu \mathrm{m}$
Figure 8. Reconstruction image

(Change sampling interval)

\subsection{Relationship between the depth of the original} object and the sampling interval of the hologram

Figure 9 and Figure 10 indicate the reconstructed image errors due to each of the sampling interval, when inclination of the original image is $0.01^{\circ}$ and $1^{\circ}$. When we compare the reproduced image error of $0.01^{\circ}$ or $0.1^{\circ}$, Reproduced image error is smaller in of $0.01^{\circ}$. From this fact, the depth of the original object and the sampling interval of the hologram are related. 


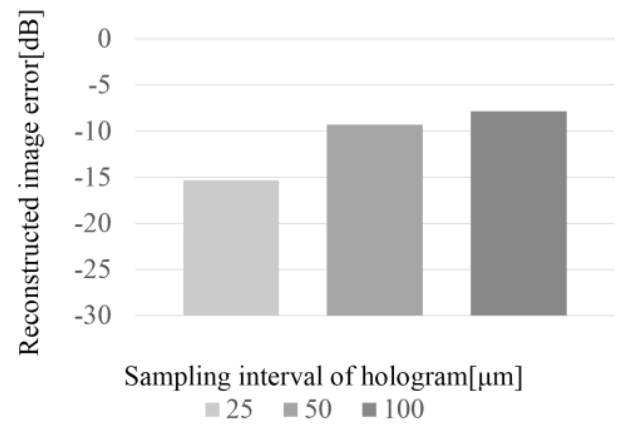

Figure 9. Reconstructed image errors (Inclination of the original image is $0.01^{\circ}$ )

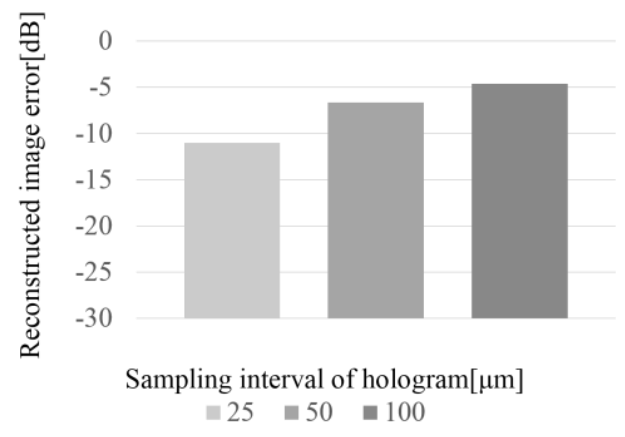

Figure 10. Reconstructed image errors (Inclination of the original image is $1^{\circ}$ )

\section{Conclusion}

Generally, in order to reconstruct the image clearly, it is necessary to use a hologram that is sufficiently large number of pixels and narrow sampling interval. However, the number of hologram pixels is limited basically, so there is a limit of the image that can be reconstructed clearly. In previous studies, the criteria for the sampling interval of the hologram and the number of sampling points necessary to reconstruct an image has not been determined. For these reasons, it is necessary to determine the criteria for the sampling interval of the hologram and the sampling points. In this paper, as its basic research, we describe the effect of the reconstructed image of $3 \mathrm{D}$ object due to a change in the hologram of the sampling interval in the computer hologram. As a result, we found that the depth of the original object and the sampling interval has relationships. What the depth of the original object is small, can be increase the sampling interval. By adjusting a sampling interval in accordance with the depth of the original object, it may be possible to reduce the number of sampling points.

\section{References}

(1) P. Hariharan : "Basics of Holography", pp. 4-12, pp. 88-89, 2004

(2) D. Leseberg, "Computer-generated three-dimensional image holograms," Appl. Opt. 31, pp.223-229, 1992

(3) H. Kim, J. Hahn, and B. Lee, "Mathematical modeling of triangle-mesh-modeled three-dimensional surface objects for digital holography," Appl. Opt. 47, pp.D117-D127, 2008

(4) Toyohiko Yatagai : "Light and Fourier transform", pp. 110-114, 1997

(5) F. Yang, A. Kaczorowski, and T. D. Wilkinson, "Fast precalculated triangular mesh algorithm for 3D binary computer-generated holograms," Appl. Opt. 53, pp. 8261-8267, 2014 